\title{
FUNCTIONAL EQUATIONS INVOLVING A PARAMETER ${ }^{1}$
}

\section{ALTMAN}

1. The present note concerns the examination of nonlinear functional equations depending on a parameter. We investigate here the iterative method described in paper [1] and [2], which is a generalization of Newton's classical method. Another abstract formalism for Newton's method has been given first by L. V. Kantorovich (for references see [4]) and applied by him to the examination of operator equations in Banach spaces.

The main point here is the application of the majorant method, which was used by Kantorovich [4] and also in paper [3].

The results stated here make it possible to find an error estimation of the exact solution in the case when the solution of a suitable approximate equation is given.

An application to approximate solutions of operator equations in Hilbert space will be given in another note.

Let $X$ and $M$ be two Banach spaces, and let $F(x, \mu)$ be a nonlinear continuous functional defined on the space $X \dot{+} M$, where $x$ and $\mu$ are in some closed spheres in $X, M$ with centres $x_{0}, \mu_{0}$, respectively.

Consider the nonlinear functional equation

$$
F(x, \mu)=0 .
$$

Let us assume that $F(x, \mu)$ is differentiable in Fréchet's sense in the spheres mentioned above with respect to each of the two variables $x, \mu$ separately. Denote by

$$
f(x, \mu)=F^{\prime}(x, \mu)=F_{x}^{\prime}(x, \mu)
$$

the partial Fréchet derivative of $F(x, \mu)$.

Putting

$$
f_{n}=f\left(x_{n}, \mu\right)=F_{x}^{\prime}\left(x_{n}, \mu\right)
$$

we choose a sequence of elements $y_{n} \in X, \mu \in M$ such that

$$
\left\|y_{n}\right\|=1, \quad f_{n}\left(y_{n}, \mu\right)=\left\|f_{n}\right\|, \quad n=0,1,2, \cdots
$$

provided that such a choice is possible.

The iterative process for solving equation (1) is defined as in papers [1] and [2]:

Received by the editors February 27, 1959.

${ }^{1}$ Based on research supported by the O.N.R., U.S.A. 


$$
\begin{gathered}
x_{1}(\mu)=x_{0}-\frac{F\left(x_{0}, \mu\right)}{f_{0}\left(y_{0}, \mu\right)} y_{0} ; \\
x_{n+1}(\mu)=x_{n}(\mu)-\frac{F\left(x_{n}, \mu\right)}{f_{n}\left(y_{n}, \mu\right)} y_{n} .
\end{gathered}
$$

Let us further assume that the second Fréchet derivative $F^{\prime \prime}(x, \mu)$ $=F_{x x}^{\prime \prime}(x, \mu)$ of $F(x, \mu)$ exists for $x$ in some sphere of $X$ with centre $x_{0}$ and that the derivatives $\partial F(x, \mu) / \partial \mu, \partial F^{\prime}\left(x_{0}, \mu\right) / \partial \mu$ and $\partial F^{\prime \prime}(x, \mu) / \partial \mu$ exist where $\mu$ belongs to some sphere of $M$ with centre $\mu_{0}$.

Consider now the real equation

$$
Q(z, \nu)=0,
$$

where $Q(z, \nu)$ is a real function of the real variables $z, \nu$, being twice continuously differentiable in the intervals $\left(z_{0}, z^{\prime}\right)$ and $\left(\nu_{0}, \nu^{\prime}\right)$. Put $Q^{\prime}(z, \nu)=Q_{z}^{\prime}(z, \nu)$ and $Q^{\prime \prime}(z, \nu)=Q_{z^{\prime}}^{\prime \prime}(z, \nu)$.

Following the argument of paper [3] let us say that equation (1) possesses a real majorant equation (4), if the following conditions are satisfied:

$$
\begin{aligned}
Q^{\prime}\left(z_{0}, \nu\right) & \neq 0 \text { and } B=-\frac{1}{Q^{\prime}\left(z_{0}, \nu\right)}>0 ; \\
\left\|F\left(x_{0}, \mu\right)\right\| & \leqq Q\left(z_{0}, \nu\right) ; \\
\frac{1}{\left\|F^{\prime}\left(x_{0}, \mu\right)\right\|} & \leqq B ;
\end{aligned}
$$

$\left(4^{\circ}\right) \quad\left\|F^{\prime \prime}(x, \mu)\right\| \leqq Q^{\prime \prime}(z, \nu)$ if $\left\|x-x_{0}\right\| \leqq z-z_{0} \leqq z^{\prime}-z_{0}$, provided that $\mu$ and $\nu$ are fixed.

The following theorem of paper [3] will be used in the sequel:

THEOREM (a). If for fixed $\mu$ and $\nu$ equation (1) possesses a real majorant equation (4), and if equation (4) has a real root $z^{*}$ in the segment $\left(z_{0}, z^{\prime}\right)$, then equation (1) has a solution $x^{*}$, where $\left\|x^{*}-x_{0}\right\| \leqq z^{\prime}-z_{0}$, and the sequence of approximate solutions $x_{n}$ constructed by process (3) converges to it. Moreover, we have the estimate

$$
\left\|x_{n}-x^{*}\right\| \leqq z^{*}-z_{n},
$$

where $z_{n}$ is defined by Newton's classical process, i.e.

$$
z_{n+1}(\nu)=z_{n}(\nu)-\frac{Q\left(z_{n}, \nu\right)}{Q^{\prime}\left(z_{n}, \nu\right)}
$$


Suppose now that the approximate solution $x_{0}$ of equation (1) is given for a certain value $\mu_{0}$ of the parameter and we are interested in the solution of this equation for some other value $\mu$ of the parameter. The following theorem concerns this case.

THEOREM 1. Let us assume that the following conditions are satisfied:

$$
\begin{array}{rlrl}
Q_{z}^{\prime}\left(z_{0}, \nu_{0}\right) & \neq 0 \text { and } B=-\frac{1}{Q^{\prime}\left(z_{0}, \nu_{0}\right)}>0 ; \\
\left|F\left(x_{0}, \mu_{0}\right)\right| & \leqq Q\left(z_{0}, \nu_{0}\right) ; & & \\
\frac{1}{\left\|F^{\prime}\left(x_{0}, \mu_{0}\right)\right\|} & \leqq B ; & & \\
\left\|F^{\prime \prime}\left(x, \mu_{0}\right)\right\| & \leqq Q^{\prime \prime}\left(z, \nu_{0}\right) & \text { if }\left\|x-x_{0}\right\| & \leqq z-z_{0} \leqq z^{\prime}-z_{0} ; \\
\left\|\frac{\partial}{\partial \mu} F\left(x_{0}, \mu\right)\right\| & \leqq \frac{\partial}{\partial \nu} Q\left(z_{0}, \nu\right) & \text { if }\left\|\mu-\mu_{0}\right\| \leqq \nu-\nu_{0} \leqq \nu^{\prime}-\nu_{0} ; \\
\left\|\frac{\partial}{\partial \mu} F^{\prime}\left(x_{0}, \mu\right)\right\| & \leqq \frac{\partial}{\partial \nu} Q^{\prime}\left(z_{0}, \nu\right) & \text { if }\left\|\mu-\mu_{0}\right\| & \leqq \nu-\nu_{0} \leqq \nu^{\prime}-\nu_{0} ; \\
\left\|\frac{\partial}{\partial \mu} F^{\prime \prime}(x, \mu)\right\| & \leqq \frac{\partial}{\partial \nu} Q^{\prime \prime}(z, \nu) & \text { if }\left\|\mu-\mu_{0}\right\| \leqq \nu-\nu_{0} \leqq \nu^{\prime}-\nu_{0}
\end{array}
$$

If equation (4) possesses a real solution $z(\nu),\left(z_{0} \leqq z(\nu) \leqq z^{\prime}\right)$, for some $\nu$, $\left(\nu_{0} \leqq \nu \leqq \nu^{\prime}\right)$, then equation (1) has a solution $x(\mu)$ if $\left\|\mu-\mu_{0}\right\| \leqq \nu-\nu_{0}$ $\leqq \nu^{\prime}-\nu_{0}$ and the sequence of approximate solutions $x_{n}(\mu)$ defined by process (3) converges to it. Moreover, we have

$$
\left\|x(\mu)-x_{0}\right\| \leqq z(\nu)-z_{0} .
$$

Proof. In order to prove this theorem it is sufficient to show that the conditions of Theorem (a) are satisfied. First of all we shall show that condition $\left(2^{\circ}\right)$ of the preceding theorem is fulfilled. In fact, we have by $\left(5^{\circ}\right)$

$$
\begin{aligned}
\left|F\left(x_{0}, \mu\right)\right| & =\left|F\left(x_{0}, \mu_{0}\right)+\int_{\mu_{0}}^{\mu} \frac{\partial}{\partial \mu} F\left(x_{0}, \bar{\mu}\right) d \bar{\mu}\right| \\
& \leqq Q\left(z_{0}, \nu_{0}\right)+\int_{\nu_{0}}^{\nu} \frac{\partial}{\partial \nu} Q\left(z_{0}, \bar{\nu}\right) d \bar{\nu} \\
& =Q\left(z_{0}, \nu_{0}\right)+Q\left(z_{0}, z\right)-Q\left(z_{0}, \nu_{0}\right)=Q\left(z_{0}, \nu\right) .
\end{aligned}
$$

Further, we get by $\left(6^{\circ}\right),\left(1^{\circ}\right)$ and $\left(3^{\circ}\right)$ 


$$
\begin{aligned}
\left\|F^{\prime}\left(x_{0}, \mu\right)\right\| & =\left\|F^{\prime}\left(x_{0}, \mu_{0}\right)+\int_{\mu_{0}}^{\mu} \frac{\partial}{\partial \bar{\mu}} F^{\prime}\left(x_{0}, \bar{\mu}\right) d \bar{\mu}\right\| \\
& \geqq\left\|F^{\prime}\left(x_{0}, \mu_{0}\right)\right\|-\left\|\int_{\mu_{0}}^{\mu} \frac{\partial}{\partial \bar{\mu}} F^{\prime}\left(x_{0}, \bar{\mu}\right) d \bar{\mu}\right\| \\
& \geqq\left\|F^{\prime}\left(x_{0}, \mu_{0}\right)\right\|\left(1-\left\|\int_{\mu_{0}}^{\mu} \frac{\partial}{\partial \bar{\mu}} F^{\prime}\left(x_{0}, \bar{\mu}\right) d \bar{\mu}\right\| \frac{1}{\left\|F^{\prime}\left(x_{0}, \mu_{0}\right)\right\|}\right) \\
& \geqq\left\|F^{\prime}\left(x_{0}, \mu_{0}\right)\right\|\left(1-\frac{\int_{\nu_{0}}^{\nu} \frac{\partial}{\partial \bar{\nu}} Q^{\prime}\left(x_{0}, \bar{\nu}\right) d \bar{\nu}}{\left\|F^{\prime}\left(x_{0}, \mu_{0}\right)\right\|}\right) \\
& \geqq\left\|F^{\prime}\left(x_{0}, \mu_{0}\right)\right\|\left(1+\frac{Q^{\prime}\left(z_{0}, \nu\right)-Q^{\prime}\left(z_{0}, \nu_{0}\right)}{Q^{\prime}\left(z_{0}, \nu_{0}\right)}\right) \\
& =\frac{\left\|F^{\prime}\left(x_{0}, \mu_{0}\right)\right\|}{Q^{\prime}\left(z_{0}, \nu_{0}\right)} Q^{\prime}\left(z_{0}, \nu\right) \geqq-Q^{\prime}\left(z_{0}, \nu\right),
\end{aligned}
$$

if the last expression is positive.

We have now to prove that $Q^{\prime}\left(z_{0}, \nu\right)$ is negative. For this purpose we shall show that $Q^{\prime \prime}(z, \nu)$ is non-negative. We have by $\left(7^{\circ}\right)$

$$
\begin{aligned}
\left\|F^{\prime \prime}(x, \mu)\right\| & \leqq\left\|F^{\prime \prime}\left(x, \mu_{0}\right)\right\|+\left\|\int_{\mu_{0}}^{\mu} \frac{\partial}{\partial \bar{\mu}} F^{\prime \prime}(x, \bar{\mu}) d \bar{\mu}\right\| \\
& \leqq Q^{\prime \prime}\left(z, \nu_{0}\right)+\int_{\nu_{0}}^{\prime} \frac{\partial}{\partial \bar{\nu}} Q^{\prime \prime}(x, \bar{\nu}) d \bar{\nu} \\
& =Q^{\prime \prime}\left(z, \nu_{0}\right)+Q^{\prime \prime}(z, \nu)-Q^{\prime \prime}\left(z, \nu_{0}\right) \\
& =Q^{\prime \prime}(z, \nu) .
\end{aligned}
$$

If $Q^{\prime}\left(z_{0}, \nu\right)$ were non-negative we should have $Q^{\prime}(z, \nu) \geqq 0$ since $Q^{\prime \prime}(z, \nu) \geqq 0$. Hence we get by $\left(1^{\circ}\right)$ and $\left(5^{\circ}\right) Q(z, \nu) \geqq Q\left(z_{0}, \nu\right) \geqq Q\left(z_{0}, \nu_{0}\right)$ $>0$. But this leads to a contradiction because equation (4) has a real solution. Thus, we conclude that condition $\left(1^{\circ}\right)$ is satisfied. It remains to prove that condition $\left(4^{\circ}\right)$ of Theorem (a) is also satisfied, i.e. $\left\|F^{\prime \prime}(x, \mu)\right\| \leqq Q^{\prime \prime}(z, \nu)$ if $\left\|x-x_{0}\right\| \leqq z-z_{0} \leqq z^{\prime}-z_{0}$, and $\left\|\mu-\mu_{0}\right\|$ $\leqq \nu-\nu_{0} \leqq \nu^{\prime}-\nu_{0}$. But this verification has already been obtained above, and thus the theorem is proved.

REMARK 1. The error estimate is given by the formula

$$
\left\|x_{n}(\mu)-x(\mu)\right\| \leqq z(\nu)-z_{n}(\nu) .
$$

This remark follows from (5).

Remark 2 . Condition $\left(2^{\circ}\right)$ can be replaced by condition 


$$
\left\|x_{1}(\mu)-x_{0}\right\| \leqq z_{1}(\nu)-z_{0} .
$$

This remarks follows from the proof of Theorem (a).

Consider now the following particular case of a functional equation depending on a parameter:

$$
F(x, \mu)=G(x)+\mu H(x)=0,
$$

where $G(x)$ and $H(x)$ are nonlinear, continuous functionals on $X$ and $\mu$ is a real number. Suppose that a solution of equation (7) is given for $\mu_{0}=0$. Applying Theorem 1 we obtain the following

THEOREM 2. Let us assume that $G(x)$ and $H(x)$ are twice continuously differentiable in the sense of Fréchet and the following conditions are fulfilled:

$$
\begin{aligned}
G\left(x_{0}\right) & =0 . \\
\frac{1}{\left\|G^{\prime}\left(x_{0}\right)\right\|} & \leqq B . \\
\left\|G^{\prime \prime}(x)\right\| & \leqq K \quad \text { if }\left\|x-x_{0}\right\| \leqq z^{\prime}-z_{0} ; \\
\left|H\left(x_{0}\right)\right| & \leqq \eta ; \\
\left\|H^{\prime}\left(x_{0}\right)\right\| & \leqq \alpha ; \\
\left\|H^{\prime \prime}(x)\right\| & \leqq \beta
\end{aligned}
$$

and

$$
\frac{(1-\alpha B \nu)^{2}}{B^{2}}-2 \nu \eta(K+\nu \beta) \geqq 0 ; \quad 0<\alpha B \nu<1 .
$$

Then equation (7) has a solution if $|\mu| \leqq \nu$ and the sequence of approximate solutions $x_{n}$ defined by process (3) converges to it. Moreover, the solution $x^{*}$ satisfies the inequality $\left\|x^{*}-x_{0}\right\| \leqq z(\nu)$ and conditions (5) and (6) hold, provided that the majorant equation (4) is replaced by the following one: ${ }^{2}$

$$
Q(z, \nu)=\frac{K+\nu \beta}{2} z^{2}-\frac{1-\alpha B \nu}{B} z+\nu \eta=0, \quad\left(z_{0}=0, \nu_{0}=0\right) .
$$

Proof. It is easy to verify that all conditions $\left(1^{\circ}\right)-\left(7^{\circ}\right)$ of Theorem 1 are satisfied.

REMARK 3. Instead of the majorant equation (8) we can use the following one ${ }^{2}$

${ }^{2}$ It seems to be interesting to notice that these majorant equations are the same as those considered by Kantorovich [4]. 
(9) $\quad Q(z, \nu)=\frac{K}{2} z^{2}+\nu \int_{0}^{z} d z_{1} \int_{0}^{z_{1}} \beta(t) d t-\frac{1-\alpha B \nu}{B} z+\nu \eta=0$.

In this case condition (6) should be replaced by condition (10)

$$
\left\|H^{\prime \prime}(x)\right\| \leqq \beta(r) \text { if }\left\|x-x_{0}\right\| \leqq r .
$$

All assertions of Theorem 2 hold if equation (9) has a positive root for $\|\mu\| \leqq \nu$.

Remark 4. Notice that Corollary 2 in $[3$, p. 23] may be considered as a particular case of Theorem 2 if we put

$$
F(x, \mu)=\left[F(x)-F\left(x_{0}\right)\right]+\mu F\left(x_{0}\right), \quad\left(\mu_{0}=1\right) .
$$

2. In this section we are concerned with the error estimation for the approximate solution of the functional equation

$$
F(x)=0,
$$

where $F(x)$ is a nonlinear continuous functional defined on the Banach space $X$.

At the same time we consider the approximate functional equation

$$
G(x)=0,
$$

where $G(x)$ is also a nonlinear continuous functional defined on $X$. Suppose that $x_{0}$ is a solution of equation (12). In order to find how near the solution of equation (11) is to $x_{0}$ we introduce the following functional equation depending on a parameter:

$$
F(x, \mu)=G(x)+\mu[F(x)-G(x)]=G(x)+\mu H(x)=0 .
$$

Suppose that both $F(x)$ and $G(x)$ are twice continuously differentiable in the sense of Fréchet. We are now in a position to apply Theorem 2. Hence we get

COROllary 1. Let us assume that the following conditions are fulfilled.

(1) $G\left(x_{0}\right)=0$,

(2) $1 /\left\|G^{\prime}\left(x_{0}\right)\right\| \leqq B$,

(3) $\left\|G^{\prime \prime}(x)\right\| \leqq K$ if $\left\|x-x_{0}\right\| \leqq z^{\prime}-z_{0}$,

(4) $\left|F\left(x_{0}\right)\right| \leqq \eta$,

(5) $\left\|F^{\prime}\left(x_{0}\right)-G^{\prime}\left(x_{0}\right)\right\| \leqq \alpha$,

(6) $\left\|F^{\prime \prime}(x)-G^{\prime \prime}(x)\right\| \leqq \beta$ if $\left\|x-x_{0}\right\| \leqq z^{\prime}-z_{0}$,

(7) $(1-\alpha B)^{2} / B^{2}-2 \eta(K+\beta) \geqq 0,(\alpha B \leqq 1)$.

Then equation (11) has a solution $x^{*}$ such that

$$
\left\|x^{*}-x_{0}\right\| \leqq z_{1} \text {, }
$$


where $z_{1}$ is the smallest root of the equation

$$
\frac{K+\beta}{2} z^{2}-\frac{1-\alpha B}{B} z+\eta=0 .
$$

This estimation may be useful especially in the case, when the expression (2) is more simple than the corresponding one for the functional $F$. We shall now apply the estimation obtained above replacing $G(x)$ by

$$
G(x)=F\left(x_{0}\right)+F^{\prime}\left(x_{0}\right)\left(x-x_{0}\right) .
$$

As the initial approach, which appears in Corollary 1, we take now the solution $x_{1}$ of equation

$$
G\left(x_{1}\right)=F\left(x_{0}\right)+F^{\prime}\left(x_{0}\right)\left(x_{1}-x_{0}\right)=0 .
$$

Condition (15), is, of course, satisfied if $x_{1}$ is defined by process (3). As a particular case of Corollary 1 we obtain

COROLLARY 2. Let us assume that the following conditions are satisfied:

(1) $\left|F\left(x_{0}\right)\right| \leqq \eta$,

(2) $1 /\left\|F^{\prime}\left(x_{0}\right)\right\| \leqq B$

(3) $\left\|F^{\prime \prime}(x)\right\| \leqq K$ if $\left\|x-x_{0}\right\| \leqq z^{\prime}-z_{0}$,

(4) $\left|F\left(x_{1}\right)\right| \leqq \eta_{1}$,

(5) $\left(1-K B^{2} \eta_{1}\right)^{2} / B^{2}-2 \eta_{1} K \geqq 0,\left(K B^{2} \eta_{1} \leqq 1\right)$.

Then equation $F(x)=0$ has a solution $x^{*}$ such that

$$
\left\|x^{*}-x_{1}\right\| \leqq z_{1},
$$

where $z_{1}$ is the smallest root of equation

$$
\frac{1}{2} K z^{2}-\frac{1-B^{2} K \eta}{B} z-\eta_{1}=0 .
$$

Let us observe that in this case the following conditions are satisfied:

(1) $G\left(x_{1}\right)=0$,

(2) $1 /\left\|G^{\prime}\left(x_{1}\right)\right\|=1 /\left\|F^{\prime}\left(x_{0}\right)\right\| \leqq B$,

(3) $\left\|G^{\prime \prime}(x)\right\|=0$,

(4) $\left|F\left(x_{1}\right)\right| \leqq \eta_{1}$,

(5) $\left\|F^{\prime}\left(x_{1}\right)-G^{\prime}\left(x_{1}\right)\right\|=\left\|F^{\prime}\left(x_{1}\right)-F^{\prime}\left(x_{0}\right)\right\| \leqq K\left\|x_{1}-x_{0}\right\| \leqq K B \eta=\alpha$,

(6) $\left\|F^{\prime \prime}(x)-G^{\prime \prime}(x)\right\|=\left\|F^{\prime \prime}(x)\right\| \leqq K=\beta$.

But this means that all conditions (1)-(7) of Corollary 1 are satisfied provided that $x_{0}$ is replaced by $x_{1}$ and $\alpha=K B \eta, \beta=K$. 


\section{REFERENCES}

1. M. Altman, On the approximate solution of non-linear functional equations, Bull. Acad. Polon. Sci. Cl. III vol. 5 (1957) pp. 457-460.

2. Concerning approximate solutions of non-linear functional equations, Bull. Acad. Polon. Sci. Cl. III vol. 5 (1957) pp. 461-465.

3. - On the approximate solutions of non-linear functional equations in Banach spaces, Bull. Acad. Polon. Sci. Ser. Math. vol. 6 (1958) pp. 19-24.

4. L. V. Kantorovich, Some further applications of Newton's method to functional equations (in Russian), Vestnik Leningrad. Univ. Ser. Mat. vol. 2 (1957) pp. 68-103.

California Institute of Techology and Academy of Science, Warsaw

\section{AN UNCOUNTABLE SET OF INCOMPARABLE DEGREES}

\section{J. R. SHOENFIELD}

The purpose of this note is to prove the following: ${ }^{1}$

THEOREM. There is an uncountable set of pairwise incomparable degrees of recursive unsolvability.

By Zorn's lemma, there is a maximal set of pairwise incomparable degrees of recursive unsolvability different from 0 ; we must show that this set is not countable. Hence our theorem follows from:

LEMmA. If $a_{0}, a_{1}, \cdots$ is a sequence of degrees different from 0 , then there is a degree $b$ which is incomparable with each $a_{n}$.

Proof. ${ }^{2}$ Let $\alpha_{n}$ be a function of degree $a_{n}$; we shall construct a function $\beta$ of degree $b$. As in [1], $\beta$ is constructed by defining inductively a function $\kappa$ such that $\kappa(a)=\bar{\beta}(\nu(a))$ with $\nu(a)=\ln (\kappa(a)) ; \kappa$ and $\nu$ must satisfy the conditions that $\kappa(a)$ is a sequence number, $\kappa(a+1)$ extends $\kappa(a)$, and $\nu(a+1)>\nu(a)$. We then have $\beta(a)=(\kappa(a+1))_{a}$ -1 .

Let $\kappa(0)=1$. To define $\kappa(a+1)$, let $n=(a)_{1}$ and $e=(a)_{2}$. If $a$ is even, set

$$
\kappa(a+1)=\kappa(a) \cdot p_{\nu(a)} \exp \left(\{e\} \alpha_{n}(\nu(a))+2\right)
$$

if $\{e\} \alpha_{n}(\nu(a))$ is defined, and $\kappa(a+1)=\kappa(a) \cdot p_{\boldsymbol{r}(a)}$ otherwise. Then clearly $\beta \neq\{e\} \alpha_{n}$ for any function $\beta$ such that $\beta(\nu(a+1))=\kappa(a+1)$.

Received by the editors April 29, 1959.

1 The problem solved in this paper was suggested to the author by C. Spector.

2 We use the notation of [1] in the proof. 